\title{
Comparación entre tres programas de enseñanza de una segunda lengua
}

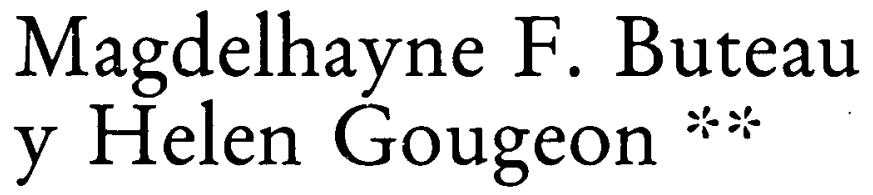

\section{Universidad McGill de Montréal}

Traducción: Seminario de Bilingüismo de la Universidad Complutense.

\section{INTRODUCCION}

Durante los últimos diez años, las materias enseñadas en francés en la escuela tradicional han sido el vehículo mediante el cual una gran parte de la población escolar anglófona de Canadá ha aprendido su segunda lengua. Esto se ha logrado mediante: (1) la asistencia a esclielas de habla francesa con la consiguiente «submersión" en la lengua y la cultura francesa, (2) la participación en los programas de inmersión en francés, lingüísticamente menos efectivos pero culturalmente más aceptables, ofrecidos dentro del contexto educativo anglófono, o (3) la selección de uno entre varios programas bilingües limitados, o en lengua francesa enriquecidos, que son accesibles generalmente a la mayoría de los estudiantes de habla inglesa.

No existe unanimidad entre los profesionales sobre la intensidad con que debe comenzar la instrucción en una segunda lengua y los efectos negativos sobre los conocimientos que son transmitidos en el idioma más débil del estudiante. La decisión sobre la aplicación de un programa en particular debe tomar en consideración el consejo de los investigadores, las metas de los padres y los problemas prácticos de la adscripción y disponibilidad del profesorado, y el desarrollo del programa.

Cada uno de los tres programas descritos en este artículo se ha iniciado como consecuencia de la insatisfacción producida por las limitaciones de los programas tradicionales de Francés como Segunda Lengua (FSL), y en respuesta a las demandas de los padres de incrementar la cantidad y calidad de francés disponible para su hijo. La edad de comienzo y la intensidad y duración de cada programa ha estado en función de ciertas consideraciones tanto teóricas como prácticas. Los informes y artículos (ver notas 1,2 y 3 ) en los que se basa este trabajo proporcionan detalles sobre la metodología, la presentación y la discusión de resultados. La intención de este trabajo es, simplemente, describir los programas, las consideraciones teóricas y prácticas que impulsaron su aplicación, resumir las tendencias de los resultados y discutir las implicaciones que tengan para el aprendizaje y la enseñanza de la segunda lengua.

\footnotetext{
* «A comparison of three second language programs». Original escrito para esta revista.

** Dirección de las autoras: McGill University, 1205 Dr. Penfield Avenue, Montréal, P2, Canadá H3A,
} $1 \mathrm{~B} 1$. 
FUNDAMENTOS DEL

DESARROLLO DEL PROGRAMA

Quèbec, una provincia en la que más del 80 por 100 de sus ciudadanos habla francés como primera lengua, ha estado promocionando de forma real el uso del francés en el comercio y la industria durante los últimos diez años. Como consecuencia, la mayoría de los padres de habla inglesa quieren que sus hijos hablen francés de modo funcional y fluido. A su juicio, $\mathrm{y}$ al de los educadores, el programa regular de francés como segunda lengua (FSL) dentro del sistema de escuela inglesa no proporciona la oportunidad de lograr tal objetivo. Por otra parte, el ir a una escuela francesa en la que el estudiante de habla inglesa pueda esperar alcanzar un alto nivel de fluidez en francés, es considerado por algunos padres como impracticable o indeseable. Consecuentemente, ha surgido la necesidad de programas alternativos de lengua francesa dentro del sector inglés, que se ajusten a las ideas actuales sobre educación bilingüe con respecto a la importancia de la edad, la capacidạd, el tiempo, la motivación y las circunstancias en el aprendizaje de la segunda lengua. La aplicación de tales programas debe tener en cuenta también las restricciones administrativas más prácticas y las características específicas de la comunidad en la que el programa va a ofrecerse.

En el caso del primer Programa Experimental, un programa innovador de Jardín de Infancia, los padres y los administradores han optado por explorar y experimentar con un programa de lengua francesa que interfiera tan poco como es posible con la instrucción regular en inglés, al mismo tiempo que se le ofrece al niño una experiencia intensa en su segunda lengua en una edad en la que, de acuerdo con los resultados de las investigaciones, está dispuesto favorablemente a la adquisición de la segunda lengua.

La selección que se ha hecho del grado cuarto para empezar el segundo Programa Experimental, está basada en la idea de que las destrezas relacionadas con la primera lengua están bien afianzadas en este momento, si bien todavía el estudiante es lo suficientemente joven como para beneficiarse de una experiencia intensiva en la segunda lengua. Se ha aplicado un razonamiento de alguna forma similar en el ter-
Inmersión en secundaria. Los muchachos que han optado por este programa son, a la vez, lo bastante jóvenes como para beneficiarse de las ventajas de su juventud en el área del aprendizaje de idiomas, y lo suficientemente mayores como para estar motivados por sí mismos y ser capaces de aprovechar al máximo un programa enriquecido de aprendizaje de la segunda lengua.

\section{COMIENZO; DURACION E INTENSIDAD DEL PROGRAMA}

La Figura 1 proporciona información sobre el grado en que se comienza, la duración y la intensidad de la instrucción en lengua francesa en los tres programas experimentales.

En el Jardín de Infancia Bilingüe, la jornada regular en lengua inglesa (sólo por la mañana), se amplía al día completo, con el fin de incluir el Programa Complementario de la segunda lengua. Este programa se centra en un cierto número de actividades interesantes en ciencias naturales y sociales. En el Programa de Continuación de los grados primero y segundo, los alumnos tienen, aproximadamente, cincuenta minutos de instrucción por día, según un programa integrado de lengua francesa y estudios sociales. En el grado tercero, cuarto y quinto, forma parte del currículum un Programa enriquecido de Francés como segunda lengua, y un Programa de estudios sociales en Francés. En el grado sexto, se le da la opción a los alumnos de ir a un Programa de Inmersión Total en Francés (es decir, todas las materias del currículum se enseñan en francés) o a un Programa Regular Inglés donde se enseña francés como segunda lengua. No se ha elaborado todavía el programa del nivel secundario, pero es probable que incluya la enseñanza de algunas materias en francés.

El segundo programa innovador de francés representado en el diagrama es el Programa de Inmersión Elemental en Francés. Después de habèr seguido un Programa Regular de Francés como Segunda Lengua desde el Jardín de Infancia hasta el grado tercero, los alumnos reciben una inmersión en el grado cuarto y quinto en un programa en el que más del noventa por ciento del curriculum se enseña en francés y, en el grado sexto, en un 
programa en el que el cincuenta por ciento del currículum está en francés, siendo sus aspectos fundamentales la instrucción en Matemáticas, Historia, Geografía y Moral y Religión. Por consiguiente, hay una reducción en el número de materias que se enseñan en francés, llegando, aproximadamente, al veinte por ciento del currículum en francés en el año de la graduación (secundaria, cinco).

El Programa de Inmersión en Francés en Secundaria se implanta en secundaria uno (grado séptimo) después de siete años (Jardín de Infancia hasta grado sexto) de haber recibido el Programa Regular de Francés como Segunda Lengua. En la secundaria uno, la instrucción de Matemáticas, Historia, Geografía y Moral y Reli- gión se realiza en francés, asi como la de otras materias. En el año de la graduación, el veinte por ciento del currículum se imparte en francés.

\section{DESCRIPCION DE LOS PROGRAMAS}

Después de haber ofrecido la panorámica general del programa en la sección anterior, proporcionamos una descripción más detallada de las principales características y los principales objetivos de los tres programas innovadores. Se incluye una breve descripción del Programa Tradicional de Francés como Segunda Lengua que siguen los estudiantes en el currículum regular inglés.

FIGURA 1

Programas experimentales

(comienzo, duración, intensidad)

JARDIN DE INFANCIA BILINGÜE Y SU CONTINUACION

INSTRUCCION EN FRANCES

INSTRUCCION EN INGLES

INSTRUCCION EN FRANCES

INSTRUCCION EN INGLES

INSTRUCCION EN FRANCES

INSTRUCCION EN INGLES

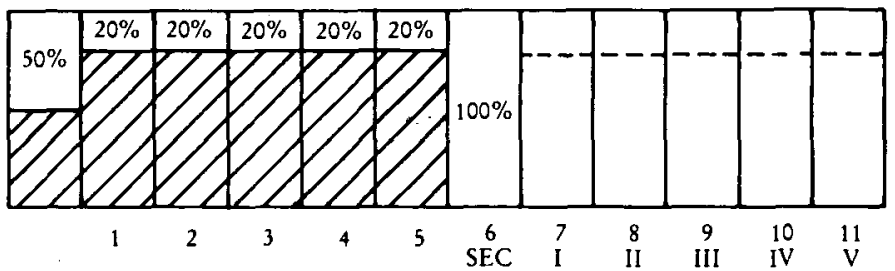

PROGRAMA DE INMERSION EN FRANCES ELEMENTAL

Fr.

L. A.

Sociales

Ciencias

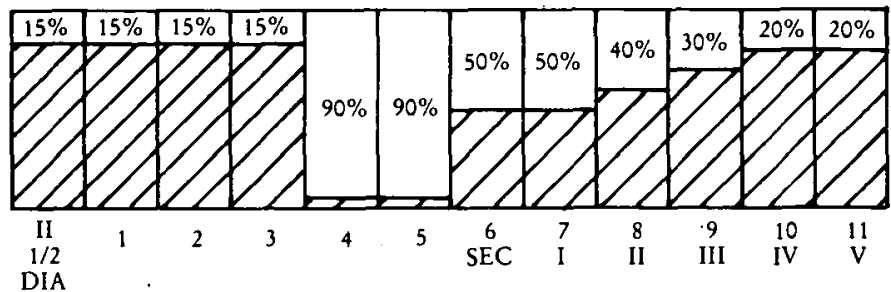

PROGRAMA DE INMERSION EN FRANCES SECUNDARIA

Fr.

L. A. Fr.

Mat. L. A

Soc. Mat. L.A.

Soc. Mat. Estudios Sociales

MRI St.

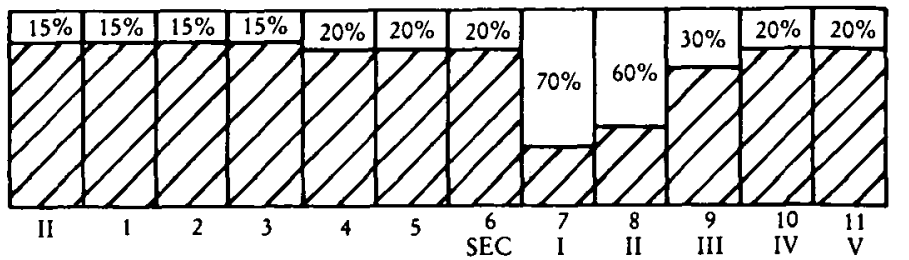


1. Francés como Segunda Lengua (Programa Tradicional)

La enseñanza del francés como segunda lengua es obligatoria en las escuelas inglesas, de acuerdo con las regulaciones provinciales, desde el grado primero al secundario cinco. Los períodos diarios varían desde veinte a treinta minutos en los seis niveles de la escuela elemental, y desde los cuarenta a cincuenta minutos en las escuelas secundarias. Se pone el acento en las destrezas orales tanto en los niveles elementales como secundarios. En el nivel secundario, estas habilidades se completan con una enseñanza sistemática de la lectura y escritura, y el programa francés comprende una variedad de opciones para adaptarse a las necesidades de los diferentes tipos de estudiantes. Además del programa de instrucción regular de la lengua francesa, los alumnos de secundaria tres, cuatro y cinco pueden optar por recibir la ensenanza de una o dos asignaturas en francés.

Generalmente, el francés es enseñado por especialistas franceses. Sin embargo, en la escuela elemental hay un cierto número de profesores encargados de la clase que tienen la obligación de impartir esta materia. Aunque gozan de una considerable libertad en la selección y uso del material, los profesores generalmente siguen las fases de las series en francés tituladas Ici on parle français de Helen Kerr, et al., y usan sus materiales y sus técnicas.

\section{Programas bilingües de Jardín de Infancia y su continuación}

El apartado francés del programa de Jardín de Infancia no repite ni revisa lo que se ha enseñado en el Jardín de Infancia inglés. El tiempo se emplea en actividades educativas agradables realizadas en francés con el fin de desarrollar la comprensión auditiva de las órdenes, comentarios y explicaciones relacionadas con los aspectos físicos y sociales de la vida escolar y la comprensión de historias cortas. Los alumnos son iniciados en la producción oral en francés de forma gradual y natural mediante canciones, poesías infantiles, juegos, etc.

Se presta una atención especial al de'sarrollo de actitudes positivas hacia el aprendizaje del francés. Se le advierte al profesor que se centre en los intereses y características de los niños y que les proporcione medios para adquirir el francés de forma natural en situaciones integradas y funcionales. Se espera que los proyectos individuales y de grupo, las visitas fuera de la escuela, el uso adecuado de materiales y medios (p. ej., vídeos TV., fragmentos de películas) proporcionen la variedad de experiencia que es necesaria para asegurarse la cooperación atenta por parte del niño durante la sesión de francés.

Los objetivos generales $\mathrm{e}$ intermedios de la parte francesa del Programa de Jardín de Infancia Bilingüe y el contenido del extracto y guía para la aplicación, se proporcionan en documentos separados (ver notas 4 y 5 , al final del artículo). El contenido del programa está basado en diez temas (p. ej., animales, salud y comida; edificios y vida comunitaria) que se desarrollan en diez módulos que están a disposición del nrofesor y se corresponden con cada uno de los meses del año escolar.

El Programa (o currículum) de Continuación consiste en la enseñanza irtegrada de Estudios Sociales y Francés como segunda lengua (FSL) en los grados primero y segundo.

El propósito de este programa experimental es mantener y reforzar las habilidades y actitudes favorables que los niños han desarrollado mediante sus trescientas horas de contacto con el profesor hablante de francés en el Jardín de Infancia.

Puesto que los alumnos han sido iniciados en el uso del francés mediante un enfoque experimental basado en actividades naturales y materiales concretos, consideramos oportuno que el Programa de Continuación adoptara un enfoque similar. La integración de los períodos diarios de Estudios Sociales y FSL parecen ofrecer un marco de referencia adecuado para este fin. Proporciona al niño la posibilidad de aprender francés en un contexto comunicativo, más intensamente, sin privarle de ningún componente del currículum regular. Además, puesto que el currículum regular de estudios sociales está diseñado para estimular la curiosidad del niño sobre las realidades cercanas a él, se espera que la integración de estas dos materias actuará como factor motivacional que haga que los sujetos disfruten aprendiendo francés mediante auténticos descubrimientos de hechos y fenómenos. Puede añadir- 
se que este tipo de enfoque integrado coincide con la práctica escolar existente que consiste en impartir estudios sociales en francés en los grados cuarto y quinto como preparación para la opción de Inmersión Total en francés en el grado sexto. Los objetivos pedagógicos generales, los objetivos finales e intermedios, así como los comentarios al programa general (syllabus) y algunas normas y mâteriales de referencia están disponibles en los documentos que aparecen al final de este artículo (notas 6 y 7 ).

\section{Programa de Inmersión en Francés, nivel elemental}

En los niveles del grado cuarto y quinto, el Programa de Inmersión en Francés está diseñado de tal forma que más del noventa por ciento de las actividades de aprendizaje puedan ser realizadas en francés. Aunque recientemente se han introducido algunas modificaciones, en las propuestas originales el tiempo de clase se distribuye como sigue:

1. El veinte por ciento para el programa central de segunda lengua, muy estructurado.

2. El veinte por ciento para cuatro centros de interés orientados hacia las matemáticas, la ciencia, los estudios sociales $y$ el arte.

3. El diez por ciento para actividades libres tales como cantar, ver televisión, etc.

4. El cuarenta por ciento para actividades centradas en un enfoque temático $o$ integrado del currículum.

La mayor parte del tiempo de instrucción está dedicado al enfoque integrado, con el fin de facilitar la interacción oral en el lenguaje objeto de aprendizaje (lo que es una meta importante de los dos primeros años del programa), y proporcionar un método de instrucción nuevo $\mathrm{y}$, afortunadamente, estimulante. La división de desarrollo del currículum del Consejo Escolar ha preparado extensos materiales utilizando un enfoque integrado. Este enfoque, que implica enseñar centrándose en un tema, se utiliza aproximadamente en el cuarenta por ciento del trabajo escolar. Para poner un ejemplo simple, el tema «bicicleta" puede usarse como medio de enseñar ciencia, matemáticas y estudios so- ciales, y como medio de aprender vocabulario y gramática. Los profesores son entusiastas de este enfoque, y se inclinan a adoptarlo en el desarrollo de sus propios materiales, menos complejos.

Como era de esperar, los primeros meses del Programa de Inmersión elemental constituyen un desafío tanto para los profesores como para los estudiantes. Los objetivos de la instrucción especifican que, hacia el final del año, el contenido del programa del grado cuarto será asimilado por la mayoría de los estudiantes y que, hacia el final del grado quinto, casi todos los estudiantes habrán logrado tal facilidad en francés que su rendimiento académico no se vería perjudicado por el uso exclusivo del francés como medio de enseñanza y aprendizaje. Por otra parte, se espera que desarrollen tal calidad de bilingüismo que su rendimiento en las habilidades de francés se aproxime gradualmente a las de los hablantes nativos franceses.

En el grado seis, los que continúan en el programa tienen aproximadamente el cincuenta por ciento de su instrucción en lengua francesa. Además del lenguaje, se dan en francés los cursos de estudios sociales y ciencias naturales. El arte, la música y la educación física se errseñan en uno u otro idioma, dependiendo de la disponibilidad del personal docente. En secundaria, el programa y el tiempo de instrucción varían en función de la escuela. En la mayoría de ellas, aproximadamente, el cuarenta por ciento del tiempo se emplea en la instrucción a través de la segunda lengua. Los estudios sociales, las ciencias $y$, en algunas escuelas, la moral, se enseñan en francés. Hay también un período dedicado a la instrucción de la lengua francesa. En secundaria dos, se implanta un programa similar.

\section{Programa de Inmersión en Francés en Secundaria}

La introducción de las clases de Inmersión en el nivel elemental acarreó gran cantidad de problemas administrativos tales como el transporte de los alumnos, la asignación de tiempos a los enseñantes, la compra y el diseño de nuevos materiales de enseñanza, y la distribución del espacio y del presupuesto. Los responsables escolares, animados por el éxito de otros. programas de inmersión iniciados en el ni- 
vel de secundaria y buscando soluciones alternativas al bilingüismo para sus alumnos, han introducido un segundo programa experimental, al comienzo de la escuela secundaria. Este programa de inmersión se implanta en secundaria. El tiempo de instrucción en francés varía desde aproximadamente el sesenta por ciento al ochenta por ciento, dependiendo de la escuela. Las matemáticas, los estudios sociales, la moral y el lenguaje se enseñan en francés. A los alumnos que asisten a su cuarto año de inmersión se les instruye separadamente de aquellos que están en su primer año de inmersión. Los alumnos de secundaria dos en su segundo año de inmersión son situados en un programa sustancialmente igual. En secundaria tres, a los estudiantes de los dos programas de inmersión, el elemental y el secundario, se les reúne para recibir instrucción en todas las materias, incluyendo una o dos que continúan dándose en francés. A los alumnos que vuelven al Programa Regular de Inglés se les dan los tradicionales cincuenta minutos de francés como segunda lengua, pero se ha diseñado un curso acelerado para ellos.

Hay más información disponible en una serie de documentos no publicados (nota 8).

\section{Otros programas}

Los estudiantes de cada uno de los tres programas experimentales que participan en las pruebas son comparados con estudiantes que siguen otros programas dentro del mismo sistema escolar o en sistemas de escuelas cercanas. A continuación se describen los programas más importantes cuyos estudiantes fueron seleccionados para esta comparación.

1) Programa Regular Inglés. Este programa se inscribe en el sistema regular inglés, en el que los estudiantes reciben aproximadamente de treinta a cuarenta minutos de instrucción en francés como segunda lengua desde el Jardín de Infancia hasta el grado sexto y, aproximadamente, cincuenta minutos después.

2) Programa Regular Francés. Programa inscrito en el sistema escolar francés. La enseñanza del inglés como segunda lengua es paralela a la enseñanza del francés dentro del programa regular en el sistema inglés. La mayoría de los estudiantes de este programa son francófonos. Los estudiantes de lengua materna inglesa que asisten a este sistema francés sufren «submersión» con respecto a la instrucción en lengua francesa.

3) Programa de recepción en Jardin de Infancia. Es un programa de Jardín de Infancia especial dentro del sistema francés que prepara a los estudiantes no francófonos para la integración con los estudiantes de habla francesa. A los efectos de esta investigación, se seleccionaron sólo aquellos estudiantes cuya lengua materna fuera el inglés, con propósitos comparativos.

\section{DISEÑO DE LA EVALUACION}

Las evaluaciones del rendimiento de los estudiantes pretenden cumplir tres metas fundamentales. La primera es evaluar el desarrollo del conocimiento que los alumnos que participan en alguno de los tres programas innovadores tienen de la lengua francesa, y, al mismo tiempo, hacer comparaciones entre los programas con respecto al rendimiento en el lenguaje objeto de aprendizaje. Una segunda meta es la evaluación de la lengua inglesa y las habilidades en los temas del área (por ejemplo, matemáticas, estudios sociales) de los alumnos en los programas experimentales, para determinar si los programas tienen efectos negativos en las destrezas en lengua inglesa o en el rendimiento en una asignatura básica. Una tercera meta es evaluar las actitudes de los alumnos hacia el programa, su percepción de su conocimiento de la segunda lengua, en relación con los alumnos de otros programas y sus actitudes hacia el aprendizaje y valoración de la segunda lengua.

Con el fin de no llegar a conclusiones basadas en resultados espúreos, se decidió seguir tres cohortes de alumnos dentro de cada uno de los tres estudios de evaluación. Dentro de cada investigación, se efectuaron comparaciones para cada cohorte, y en cada grado entre los alumnos que asistían al programa experimental y los que atendían a los programas con los que se comparaban, tanto en la misma escuela como en escuelas vecinas.

Para cada cohorte de los Programas de Inmersión Elementales y Secundarios se seleccionó una muestra al azar, por escuela, al comienzo de los programas (grado 
cuarto y grado séptimo) para cada uno de los grupos de comparación. Para cada cohorte, las poblaciones tenían que ser remuestreadas al comienzo de la secundaria, donde aproximadamente el 40 por 100 de los alumnos que, en este momento, estaban en el programa inglés regular, escogieron entrar en el Programa de Inmersión en Francés, en secundaria. Se aplicaron tests aproximadamente a trescientos alumnos en el grado cuarto, en el grupo experimental. En el grado octavo, con la introducción del segundo Programa de Inmersión en Francés, la muestra se redujo a aproximadamente 150 . En cada uno de los grados se incluyó en la muestra del 15 al 20 por ciento de la población.

En el caso de los Programas de Jardín de Infancia Bilingüe y su continuación, se aplicaron los tests a una población total de una clase y se compararon con los grupos de comparación (clases) de un status socioeconómico similar.

La naturaleza voluntaria del programa en cada nivel no permitía una ordenación aleatoria de los alumnos en los tratamientos (programas). Consecuentemente, debido a la probabilidad de diferencias iniciales entre los grupos experimentales y de comparación y a la movilidad de las muestras debido a que los alumnos dejaban el programa por varias razones, la mayoría de los tests fueron administrados al comienzo y al final de cada año y, cuando fue posible, se utilizaron diseños de análisis de covarianza como tratamiento estadístico de los datos. En la mayoría de los casos, se aplicó el Test de Matrices Progresivas de Raven o el Test Canadiense
Lorge Thorndike para ajustar las medidas del test de rendimiento a cualesquiera diferencias entre las muestras en aptitud general.

Se ha aplicado seis categorías de tests, incluyendo tests de aptitud mental, tests de contenidos (p. ej., matemáticas, estudios sociales), tests de conocimiento del inglés, tests de conocimiento del francés, técnicas de observación de clase y cuestionarios de actitudes (alumnos, padres, profesores). Estos tests cubrían un amplio espectro, incluyendo estandarizados y no estandarizados, individuales y colectivos, orales y escritos.

Los tests fueron administrados y puntuados por estudiantes postgraduados entrenados, que fueron supervisados, en todos los aspectos de estas operaciones, por las dos investigadoras de la universidad. Los tests abiertos fueron puntuados por el método ciego y de forma independiente por dos puntuadores, y se calcularon medidas de fiabilidad interjueces. Todas las interpretaciones de resultados y la subsiguiente redacción de artículos e informes fueron dirigidos por las investigadoras principales.

En la Tabla 1 se ofrece una lista de los tests administrados a los estudiantes en los grupos experimentales y de comparación. Los instrumentos no estandarizados fueron desarrollados, puestos a prueba y validados por las investigadoras. En los informes anuales está disponible una descripción de estos tests y, cuando era apropiado, los procedimientos de puntuación y validación. 
TABLA 1

Tipos de test:

\begin{tabular}{|c|c|c|}
\hline Jard. Inf. Bilingüe & Inmersión elemental & Inmersión secundaria \\
\hline (I Grados $1,2,3,4$ ) & (Grados 4, 5,6) & (Grados 7, 8, 9, 10,11) \\
\hline \multirow{3}{*}{$\begin{array}{l}\text { ESTANDARIZADOS } \\
\text { Raven } \\
\text { Peabody Picture Vocabulary } \\
\text { Bilingual Syntax Measure } \\
\text { Metropolitan Achiev. Tests }\end{array}$} & ESTANDARIZADOS & ESTANDARIZADOS \\
\hline & $\begin{array}{l}\text { Raven } \\
\text { Lorge-Thorndike } \\
\text { Test Canadiense de apt. basic. }\end{array}$ & $\begin{array}{l}\text { Raven } \\
\text { Lorge-Thorndike } \\
\text { Metropolitan Achievement Tests }\end{array}$ \\
\hline & $\begin{array}{l}\text { Vocabulario } \\
\text { Lectura }\end{array}$ & $\begin{array}{l}\text { Conocimiento del mundo } \\
\text { Lectura }\end{array}$ \\
\hline \multirow{3}{*}{$\begin{array}{l}\text { Lectura } \\
\text { Lenguage } \\
\text { Comprens. Franc. (OISE) } \\
\text { Comprens. Lect. Francés (OISE) }\end{array}$} & $\begin{array}{l}\text { Ortografia } \\
\text { Mayúsculas }\end{array}$ & $\begin{array}{l}\text { Lenguaje } \\
\text { Ortografie }\end{array}$ \\
\hline & $\begin{array}{l}\text { Puntuación } \\
\text { Uso del leng. } \\
\text { Matematic. }\end{array}$ & $\begin{array}{l}\text { Mat. } 1 \text { (Operaciones) } \\
\text { Mat. } 2 \text { (Concepto) } \\
\text { Mat. } 3 \text { (Problemas) }\end{array}$ \\
\hline & $\begin{array}{l}\text { Je Sais (Test de Compr. Audit.) } \\
\text { Test de Rendim. en franc. } \\
\text { Tests de Francés (CECM) } \\
\text { Prueba de lectura silenciosa } \\
\text { Tests de lectura "California" } \\
\text { Tests de comprensión (IOPF) }\end{array}$ & $\begin{array}{l}\text { Je Sais } \\
\text { Tests de Rendim. en franc. } \\
\text { Tests de Français (CECM) } \\
\text { Prueba de lectura silenciosa } \\
\text { Prueba de lectura rápida (SLP) } \\
\text { Tests de lectura "California" }\end{array}$ \\
\hline NO ESTANDARIZADOS & NO ESTANDARIZADOS & NO ESTANDARIZADOS \\
\hline $\begin{array}{l}\text { Peabody Picture Voc. (Fran.) } \\
\text { Escala de Sintax Bilingue (ESB) } \\
\text { Medida Leng. Oral (Inq.\&Fr) } \\
\text { Test Compr: Lect. Fran. Buteau-Gougeon } \\
\text { Contenido (en francés) } \\
\text { Análisis de interacción verbal } \\
\text { Cuestionario de actitudes de los alumnos } \\
\text { Cuestionario de profesores } \\
\text { Cuestionario de padres }\end{array}$ & $\begin{array}{l}\text { Comprensión y Expresión Oral } \\
\text { en Franc (Buteau-Gougeon) }\end{array}$ & $\begin{array}{l}\text { Medida de leng. oral en francés } \\
\text { Cloze ingl. } \\
\text { Cloze fran. } \\
\text { Expresión escrita ingl. } \\
\text { Expresión escrita fran. } \\
\text { Entrevista lenguaje oral en fran. } \\
\text { (analizada según contenido y lenguaje) } \\
\text { Cuestionario de actitudes de los estudiantes. }\end{array}$ \\
\hline
\end{tabular}

* Los tests incluidos no fueron empleados en todos los grados. 
TABLA 2

Organización de las aplicaciones longitudinales

\begin{tabular}{|c|c|c|c|c|c|c|c|c|c|c|c|c|}
\hline Nivel & $1972-73$ & $1973-74$ & $1974-75$ & $1975-76$ & $1976-77$ & $1977-78$ & $1978-79$ & $1979-80$ & $1980-81$ & $1981-82$ & $1982-83$ & $1983-84$ \\
\hline II & & & & & & & & Co. I & Co. II & Co. III & & \\
\hline 1 & & & & & & & & & Co. I & Co. II & Co. III & \\
\hline 2 & & & & & & & & & & Co. 1 & Co. II & Co. III \\
\hline 3 & & & & & & & & & & & Co. 1 & Co. II \\
\hline 4 & $\mathrm{Co}_{\text {elem }} \mathrm{I}$ & $\mathrm{Co}_{\mathrm{elem}} I I$ & $\mathrm{Co}_{\text {ctem }}$ III & & & & & & & & & Co. I \\
\hline 5 & & $\mathrm{Co}_{\mathrm{elem}} \mathrm{I}$ & $\mathrm{Co}_{\mathrm{clcm}} \mathrm{II}$ & $\mathrm{Co}_{\text {clem }} 111$ & & & & & & & & \\
\hline 6 & & & $\mathrm{Co}_{\text {elem }} \mathrm{I}$ & $\mathrm{Co}_{\mathrm{clcm}} \mathrm{II}$ & $\mathrm{Co}_{\mathrm{clcm}} \mathrm{III}$ & & & & & & & \\
\hline 7 & & & & $\begin{array}{l}\mathrm{Co}_{\mathrm{sltum}} \mathrm{I} \\
\mathrm{Co}_{\mathrm{scc}} \mathrm{I}\end{array}$ & $\begin{array}{l}\mathrm{Co}_{\mathrm{clcm}} \mathrm{II} \\
\mathrm{Co}_{\mathrm{rcc}} \mathrm{II}\end{array}$ & $\begin{array}{l}\mathrm{Co}_{\mathrm{etem}} \text { III } \\
\mathrm{Co}_{4 \mathrm{i}} \text { III }\end{array}$ & & & & & & \\
\hline 8 & & & & & $\begin{array}{l}\mathrm{Co}_{\mathrm{vikm} \mathrm{I}} \mathrm{I} \\
\mathrm{Co}_{\mathrm{ke} \mathrm{I}} \mathrm{I}\end{array}$ & $\begin{array}{l}\mathrm{Co}_{\mathrm{clum}} \mathrm{II} \\
\mathrm{Co}_{\mathrm{sec}} \mathrm{Il}\end{array}$ & $\begin{array}{l}\mathrm{Co}_{\mathrm{elm}} \mathrm{III} \\
\mathrm{Co}_{1 \mathrm{ic}} \mathrm{Ill}\end{array}$ & & & & & \\
\hline 9 & & & & & & $\begin{array}{l}\mathrm{Co}_{\mathrm{cllem} \mathrm{I}} \\
\mathrm{Co}_{\mathrm{sic}} \mathrm{I}\end{array}$ & $\begin{array}{l}\mathrm{Co}_{\mathrm{ekm}} \mathrm{Il} \\
\mathrm{Co}_{4 \mathrm{II}} \mathrm{II}\end{array}$ & $\begin{array}{l}\mathrm{Co}_{\mathrm{ck} \mathrm{km}} \mathrm{III} \\
\mathrm{Co}_{\mathrm{si}} \text { III }\end{array}$ & & & & \\
\hline 10 & & & & & & & $\begin{array}{l}\mathrm{Co}_{\mathrm{clem}} \mathrm{I} \\
\mathrm{Co}_{\mathrm{wi}_{\mathrm{i}} \mathrm{I}}\end{array}$ & $\begin{array}{l}\mathrm{Co}_{\mathrm{ckm}} \mathrm{Il} \\
\mathrm{Co}_{\mathrm{scc}} \mathrm{II}\end{array}$ & $\begin{array}{l}\mathrm{Co}_{\mathrm{ck} \mathrm{mm}} \mathrm{Ill} \\
\mathrm{Co}_{\mathrm{sec}} \mathrm{lll}\end{array}$ & & & \\
\hline 11 & & & & & & & & $\begin{array}{l}\mathrm{Co}_{\mathrm{cl} \mathrm{m} m} \mathrm{I} \\
\mathrm{Co}_{\mathrm{stc}} \mathrm{I}\end{array}$ & $\begin{array}{l}\mathrm{Co}_{\mathrm{clem}} \mathrm{II} \\
\mathrm{Co}_{\mathrm{stc}} \mathrm{Il}\end{array}$ & $\begin{array}{l}\mathrm{Co}_{\mathrm{kl} \cdot \mathrm{m}} \mathrm{III} \\
\mathrm{Co}_{\mathrm{sk}} \mathrm{Ill}\end{array}$ & & \\
\hline
\end{tabular}


RESUMEN GENERAL DE LOS RESULTADOS DE LOS TESTS

En los apéndices a y b (Resultados) se ofrece un resumen de las tendencias generales en los resultados de los tres estudios, en una selección de tests de lenguaje en inglés y en francés, y de medidas actitudinales. Cuando se indican diferencias entre grupos de comparación, las diferencias entre las medidas de los grupos son, en la mayoría de los casos, estadísticamente significativas. Se remite al lector a los informes anuales para una presentación más precisa de los resultados dentro de cada cohorte.

Basándonos en las tendencias globales de los resultados, hemos llegado a las conclusiones generales que se exponen a continuación. con referencia a los hallazgos provenientes de la evaluación del Programa Bilingüe de Jardín de Infancia y de los dos Programas de Inmersión en Francés:

\section{Programas de Jardín de Infancia.y su continuación}

1. En el nivel de Jardín de Infancia no hay diferencias estadísticamente significativas entre los grupos indicados en el Apéndice $D$, en cualquiera de los tests de lenguaje. Aparecen diferencias en la dirección esperada en las medidas de lengua francesa. Generalmente, los alumnos en el Jardín de Infancia Regular en Francés (RF) logran resultados significativamente mejores que los del Jardín de Infancia de Recepción $(R)$, quiénes, a su vez, logran mejores resultados que los que están en el Jardín de Infancia Bilingüe Experimental (Exp.). En la mayor parte de los casos, las diferencias entre el Jardín de Infancia de Recepción y el Jardín de Infancia Bilingüe no son significativas.

2. Se observa un patrón general similar al anterior en el grado primero. De nuevo, no hay diferencias intergrupo significativas en los tests de inglés, mientras que en los tests de francés las diferencias entre los grupos (Recepción, Experimental, Inglés Regular) se producen en la dirección esperada. Aunque las diferencias en comprensión en francés indican que el grupo de post-recepción tiene una ventaja sobre el grupo experimental, no hay diferencias significativas en las medidas de francés oral.
3. En los grados segundo y tercero, el grupo de Jardín de Infancia de Recepción sobrepasa al grupo Experimental y al grupo de Inglés Regular en los tests de francés. El grupo Experimental mantiene su adelanto sobre el grupo de Inglés Regular en los tests de francés. Además, en el área de estudios sociales, el grupo Experimental no ha sufrido ningún detrimento en su conocimiento de contenidos cuando se le compara con los otros grupos.

4. Los resultados de un cuestionario de actitudes administrado en el cuarto grado revela una voluntad de utilizar el francés fuera de la escuela en situaciones que justifique su uso (p. ej., en las compras). La identificación positiva con la lengua y la cultura francesa no es tan visible como se había supuesto previamente.

5. Desde la perspectiva de los padres, el programa se evaluó como ventajoso, y casi todos los padres lo recomendaron a otros. Los profesores que parciciparon en la aplicación de los programas señalaron deficiencias específicas, particularmente en el área de la clasificación de ciertos objetivos lingüísticos, pero son de la opinión de que los programas son viables y pueden mantenerse.

\section{Programas de Inmersión en Francés en Elemental y Secundaria}

1. Si nos basamos en los resultados de los tests de aptitud y en los pretests de inglés y matemáticas, parece que el Programa de Inmersión atrae más a los estudiantes académicamente más capaces y menos a los estudiantes académicamente menos capaces. Las puntuaciones pretests (datos de línea base para cada una de las cohortes) favorecen consistentemente a los grupos de inmersión. Los estudiantes que dejaban el Programa de Inmersión durante el primer año son con frecuencia aquellos que experimentaban dificultades, lo que reducía la heterogeneidad de la muestra.

2. En el nivel elemental, la participación en la inmersión se traduce en algunos efectos temporales sobre el rendimiento en los tests de inglés. En el comienzo del grado cuarto, previo a la participación en el Programa de Inmersión, la muestra de Inmersión en cada cohorte logró medias en los subtests que eran significativamente mayores que las obtenidas por la muestra de Inglés Regular. Al final del grado cuar- 
to $\mathrm{y}$ al principio del grado quinto, las dos muestras eran equivalentes en sus resultados. En algunas destrezas específicas de inglés (p. ej., deletrear, mayúsculas, puntuación), las diferencias significativas entre las medias favorecían a la muestra de Inglés Regular. La participación de los estudiantes de inmersión en el Programa de $\mathrm{mitad} / \mathrm{mitad}$ en el nivel sexto, se traducía en volver a ganar la posición más dominante mantenida por este grupo al comienzo del grado cuarto. En el nivel secundario, las diferencias en las destrezas, en inglés, entre los estudiantes en el Programa de Inmersión en Secundaria y aquellos que estaban en el Programa de Inglés Regular eran menos marcadas. El hecho de que, en el Programa de Inmersión en Secundaria, los estudiantes siguieran aproximadamente el 35 por 100 del currículum en inglés, podía ser la causa de que se mantuviera la habilidad lingüistica en el nivel de sus equivalentes dentro del Programa Regular.

3. En los tests estandarizados de destrezas en francés, los resultados del análisis estadístico de las puntuaciones medias revela que al final de Secundaria tres, los estudiantes que optaron por el Programa de Inmersión Secundaria en Francés habían alcanzado aproximadamente el mismo nivel de rendimiento que los estudiantes que entraron en el de Inmersión en Francés en el grado cuarto, incluso aunque las tendencias más diferenciales favorecieran al último grupo. En los tests no estandarizados de francés oral y expresión escrita, hay una tendencia consistente que revela que el grupo de Inmersión Elemental está por delante del grupo de Secundaria.

4. En el cuestionario de actitudes, los estudiantes de Inmersión Elemental (secundaria cinco) puntuaban más alto en rendimiento en francés que los estudiantes de Inmersión Secundaria. Con respecto a la selección de los programas preferidos, un porcentaje mayor de estudiantes de inmersión postelemental parecían más satisfechos con sus propios programas que los de inmersión postsecundaria o que los de Programas Regulares. Los sujetos que contestaban, no creían que la instrucción en la lengua más débil tuviera efectos negativos sobre el éxito académico. Consideraban algunas materias (p. ej., la historia y la geografía) como más apropiadas que otras (p. ej., las ciencias, las matemá- ticas) a la hora de ser incluidas en los Programas de Inmersión.

\section{ALGUNAS IMPLICACIONES PARA LA ENSEÑANZA Y EL APRENDIZAJE}

A continuación exponemos algunas observaciones personales formuladas como consecuencia de las observaciones de clase y los resultados de la evaluación longitudinal de un programa implantado a tres niveles.

\section{Programas de Jardín de Infancia Bilingüe y su continuación}

Dado que la meta principal de este programa (Jardín de Infancia; grados primero y segundo) era simplemente proporcionar una experiencia rica y temprana en francés, con el fin de desarrollar actitudes más positivas hacia el uso de la lengua y una mayor facilidad en comprensión y expresión que la que proporciona un programa regular FSL, este programa puede considerarse un éxito. Puesto que el programa, después del Jardín de Infancia y hasta la Inmersión Total en el grado sexto afecta sólo a una pequeña parte de la jornada escolar, es necesario que se implante un FSL enriquecido desde los grados tercero al quinto, de forma que los resultados positivos de la experiencia temprana e intensa en francés puedan tener una continuidad.

\section{Programas de Inmersión en Francés, Elemental y Secundario}

La opción de acudir a cualquiera de los Programas de Inmersión en Francés se ofrece a todos los alumnos en cualquier distrito escolar importante, pero se produce de hecho un sesgo de manera indirecta, puesto que la mayoría de los estudiantes más capaces seleccionan un programa experimental, lo que puede llevar a una situación donde las clases que sigan el Programa Regular estén compuestas de un número anormalmente alto de estudiantes académicamente más flojos. Si los Programas Regulares quedan como una opción viable dentro del sistema, es necesario ofrecer una atención especial a los alumnos en esos programas.

Basándonos en los datos proporciona-: 
dos por los resultados en los tests de lectura en francés, los de expresión escrita libre y las medidas de lenguaje oral, hemos identificado una especie de interlengua$j e »$ en algunos alumnos. Con el fin de que pueda facilitarse la adquisición de una competencia similar a la de los nativos, se recomienda una exposición más amplia a la segunda lengua, y un entrenamiento sistemático en ciertos aspectos del francés escrito. Esto puede lograrse mediante un curso bilingüe de «competencias de lenguaje» especial, que puede incorporar el estudio comparativo de las características del inglés y del francés, y también estrategias de desarrollo del vocabulario y técnicas de comunicación general que pueden llevar a un nivel muy alto el uso de ambos idiomas.

Es imprescindible que un programa de mantenimiento incluya cierta cantidad de experiencias con la lengua en la comunidad de habla francesa, de tal forma que los estuciantes tengan la oportunidad de aumentar sus destrezas orales y exponerse a una variedad de modelos en su segunda lengua.

En vista de los informes favorables so- bre la inmersión en todo Canadá, y de nuestros propios hallazgos en este área y en el Programa Bilingüe Temprano, es necesario apoyar la aplicación de los programas que proporcionen una práctica pedagógica con más éxito de la enseñanza del francés que la que proporciona el currículum tradicional. Este hallazgo es el más claro, sobre todo cuando va acompañado de resultados que indican la ausencia de efectos negativos a largo plazo en las destrezas de la lengua nativa y de efectos perjudiciales pequeños o inexistentes sobre el aprendizaje de las materias tradicionales que se enseñan en francés. Aunque los hallazgos de la presente investigación corroboran los de otros estudios, podríamos plantearnos algunas cuestiones sobre el desarrollo académico y cognitivo de los estudiantes a los que se les enseña asignaturas como Ciencias e Historia a través de su lengua más débil y sobre las destrezas lingüísticas de los estudiantes cuyo currículum está centrado más en el contenidú que en el lenguaje. El intento de responder a estas críticas cuestiones va más allá del alcance de este trabajo y de las metas de la presente evaluación.

\section{Notas}

${ }^{1}$ BUteaU, M. F., y Gougeon, H. Five annual progress reports and one summary report on the evaluation of the bilingual kindergarten and follow-up programs, St. Croix School Commission 1979-1984.

2 BUTEAU, M. F., y GOUGEON, H.: Six progress reports and one summary report on the evaluation of the Elementary and Secondary Immersion Programs, Lakeshore School Commission 1972-1983. ${ }^{3}$ Publicaciones y conferencias relacionadas con la evaluación de los tres programas innovadores:

-: BUTEAU, M. F., y GoUgEON, H.: Tip of the Iceberg on French Immersion. Comunicación presentada en la Canadian Learned Societies Conference, Toronto, junio 1974, págs. 15.

-: A Balance Sheet: Report on a longitudinal evaluation of two parcial French immersion program. Comunicación presentada en la Canadian Learned Societies Conference, Londres, junio 1978, págs. 20.

-: Two French immersion programs: A longitudinal evaluation of student achievement on English and French language tests. Comunicación presentada en la Eastern Educational Research Association, Norfolk, Virginia, marzo, 1980, pág. 14.

-: An Analysis of the Opinions of Three Groups of Secondary Five Students on Their Study of French. Comunicación presentada en la Canadian Learned Societies Conference, Vancouver, 1984.

-: The use of the cloze procedure in evaluating global language proficiency in bilingual contexts. Comunicación presentada en la the Third Annual Conference of the Teaching of Reading in Bilingual Education, Texas Women's University, Denton, Texas, June, 1984, pág. 28.

-: The Assessment of some Aspects of Verbal Behaviour in a Bilingual Kindergarten Program, en M. Lutjeharms y T. Culhane (eds.) Practice and Problems in Language Testing, Brussels, Belgium, Vrije Universiteit Brussel, 1982, 193-203.

-: BUTEAU, M. F., y GoUgeON, H. l'Evaluation de la compréhension du français oral chez les élèves d'origine portugaise inscrits dans les classes d'accueil et de francisation. Direction générale des réseaux, Ministère de L'Education, mayo, 1982.

-: Le test de closure en langue premiére et en langue seconde: son utilisation dans l'evaluation de différents programmes d'immersion. Mesure et Evaluation en Education, vol. 5, 4-15 (versión en inglés disponible).

-: Maternelle Bilingue: Evaluation Longitudinale des progrès en français langue seconde, Les Annales de'ACFAS, 1983, 50, no 3 (versión en inglés disponible).

-: Enseignement du français par immersion partielle - Analyse D'observations. Les Annales de l'ACFAS 1985 , vol. 51 , no 1 (versión en inglés disponible).

4 Buteau, M. F., y Gougeon, H.: Programme expérimental d'enseignement bilingue à la maternelle, soumis au directeur général, Direction générale du développement pédagogique, le 26 février 1979. 
${ }^{5}$ BUteau, M. F., y Gougeon, H.: Programme expérimental d'enseignement bilingue à la maternelle, soumis au directeur général. Direction générale, du développement pédagogique, le $1^{e r}$ février, 1980 .

${ }^{6}$ BUTEAU, M. F., y GOUGEON, H.: Programme expérimental d’enseignement intégré des sciences humaines et du français langue seconde en première année, préparé pour la Commission scolaire Sainte-Croix, 1980.

${ }^{7}$ Buteau, M. F., Y Gougron, H.: Programme expérimental d'enseignement intégré des sciences humaines et du français langue seconde en deuxième année, préparé pour la Commission scolaire SainteCroix, 1981.

${ }^{8}$ En la División de los Servicios de Desarrollo curricular (Curriculim Development Services Division) del Lakeshore School Board, Lakeshore, Québecestán disponibles una serie de documentos y artículos sin publicar referentes a las metas y objetivos de los Programas de Inmersión en francés Elemental y Secundaria.

\section{Resumen}

En este artículo se describen tres programas de educación bilingüe, cuya aplicación se ha realizado como resultado de la falta de satisfacción existente con los programas tradicionales de Franceś como Segunda Lengua, y como respuesta a las demandas de los padres para incrementar la cantidad y calidad del francés a que estaban expuestos sus bijos. En el artículo se proporciona información sobre el momento en que comienza la instrucción en francés, su duración y su intensidad en los tres programas experimentales. Se describe cada programa, incluyendo el tradicional de Francés como Segunda Lengua. Se incluye la evaluación de los programas y una revisión general de los resultados, a partir de los cuales se hacen algunas reflexiones sobre la enseñanza y el aprendizaje de una segunda lengua.

\section{Summary}

Three programs of bilingual education are described in this paper. These programs were initiated as a result of dissatisfaction about the limitations of traditional French as a Second Language (FSL) programs and in response to parental demands to increase the quantity and quality of French available to their children. Information is provided on the grade of onset, duration and intensity of the French as a Second Language (traditional Progam). Design of evaluation and an overview of test results from which some implications for teaching and learning a second language were derived, are also included.

\section{Résumé}

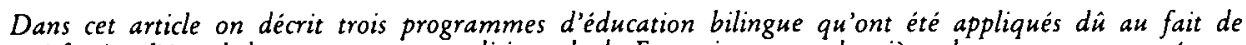
l'insatisfaction l'égard des programmes traditionnels de Français comme deuxième langue et comme réponse à la demande des parents pour augmenter la quantité et qualité du Français dans les écoles.

Information sur le moment auquel on commence l'instruction en Français, sa duration et l'intensité des trois programmes expérimentaux est donnée ainsi que du programme traditionel du Français comme deuxième langue.

On dècrit en suite l'évaluation et l'aperçu générale des résultats á partir desquels on dégage quelques réflexions sur l'enseignement et l'apprentissage d'une deuxième langue. 


\section{DESCRIPCION DE LOS INSTRUMENTOS NO ESTANDARIZADOS UTILIZADOS EN LA EVALUACION DE LOS TRES PROGRAMAS INNOVADORES DE LENGUA FRANCESA}

\section{A) Programas Bilingües de Jardín de Infancia y su continuación}

\section{Test de Vocabulario Gráfico de Peabody Peabody Picture Vocabulary Test) Versión francesa.}

La versión inglesa de este test proporciona una estimación de la inteligencia verbal del niño mediante la medida del vocabulario que entiende. Las normas están basadas en los resultados de niños entre dos años y medio y dieciocho años. El test consiste en una serie de 150 láminas, conteniendo cada una de ellas cuatro dibujos. El sujeto debe indicar, para cada conjunto de cuatro dibujos, aquel que se corresponde mejor con la palabra presentada por el examinador.

La versión francesa de este test consiste en una traducción adaptada para ser utilizada con niños francófonos. Las reglas para la administración son las mismas que las indicadas en la versión inglesa. La puntuación directa está basada en el número de respuestas correctas antes de que el niño cometa seis errores en ocho items consecutivos. La versión francesa no está estandarizada.

La versión inglesa del Peabody fue administrada a los alumnos en el Jardín de Infancia Bilingüe y en los grados primero y segundo (su continuación), y en el Jardín de Infancia Inglés y eĩ lós gráuos primero y segundó de la escuela inglesa; la versión francesa, en el jardin de Íntancia Francés y en el Jardín de Infancia Bilingüe y los grupos correspondientes de los grados primero y segundo.

\section{La escala de sintaxis bilingüe (L'echelle de syntaxe bilingue) (E.S.B.)}

Esta versión francesa del Bilingual Syntax Measure ha sido desarrollada por Paul Pimsleur y usada por su colaboradora en la Universidad de París III, Elisabeth Bautier-Castaing, en su investigación sobre la adquisición del francés.

La ESB, que usa los mismos dibujos que la BSM (versión inglesa) consta de 34 preguntas y se refiere a 56 estructuras sintácticas. A diferencia de la versión original, la ESB no permite, todavía, el cálculo de un nivel preciso de competencia.

En vista del número limitado de sujetos y del número restringido de estructuras sintácticas emitidas, se ha desarrollado una adpatación del procedimiento de Bautier-Castaing y se ha realizado un análisis simplificado. Reagrupando ciertas estructuras, el análisis está basado en parámetros tales como: frases gramticalmente aceptables, género, el verbo ser y el verbo tener, verbos regulares e irregulares (presente), etc.

\section{Medida del lenguaje oral (Oral Language Measure) (OLM)}

El Test de Lenguaje Oral consiste en una entrevista de aproximadamente diez minutos sobre los acontecimientos representados en una serie de dibujos. El tema de una de las series es la celebración de una fiesta familiar de cumpleaños. Este ejercicio de expresión oral es parcialmente estructurado y parcialmente libre. Implica la descripción de los dibujos, la formulación de posibles conversaciones entre los personajes representados en esos dibujos, relacionándolas con la historia total, y la explicación de un acontecimiento específico representado en una de las escenas.

En la versión francesa se empleó una escala de 0 a 5 para cada uno de los tres siguientes parámetros: comprensión del acontecimiento representado en las series; desarrollo léxico y gramatical; nivel discursivo y enunciativo de la producción total. Se utilizó una escala de 0 a 3 para puntuar la capacidad para la expresión personal a la hora de explicar la percepción de un acontecimiento. Además, se contaron las palabras en inglés y francés basándose en una transcripción escrita de las respuestas grabadas. En línea con una evaluación más restringida de las destrezas en lengua inglesa, y usando la misma escala, se valoran la calidad de la comunicación en la versión inglesa basándose en las puntuaciones del desarrollo léxico y del nivel lingǘstico general (incluyendo nivel gramatical, discursivo $e$ innovador). 
Bilingüe y su continuación, y de los grados primero y segundo, así como a los alumnos del Jardín de Infancia de acogida y de los grados primero y segundo del sistema francés. La versión francesa se administró a los alumnos de los grupos del Jardín de Infancia Francés y del Jardín de Infancia de Acogida y de los grados primero y segundo de postacogida.

\section{El Test de Comprensión Lectora en Francés Buteau-Gougeon}

Este texto fue diseñado para evaluar la comprensión lectora en francés de los estudiantes de habla inglesa de ocho a diez años de edad que habían adquirido un buen nivel de comprensión oral en francés mediante las lecciones de Estudios Sociales recibidas en este idioma. Consiste en un texto seguido por una serie de preguntas. Se llegó a la forma final después de varios ensayos con estudiantes francófonos y anglófonos.

El texto está formado por cuatro párrafos e incluye aproximadamente 350 palabras. Es una versión adaptada de un pasaje sobre una escena de invierno de una lectura francesa. Las preguntas consisten en ítems de elección múltiple (9) e ítems de comprensión (5). Según el contenido, las preguntas tratan de evaluar la comprensión léxica (4), inferencial (8) y evaluativa (2).

Los alumnos tienen la oportunidad de ser iniciados en este tipo de test, con la ayuda del examinador, mediante un ejercicio de práctica sobre un corto pasaje, seguido de una pregunta de elección múltiple y una pregunta de comprensión.

Después, se les da a los alumnos veinticinco minutos para leer el test y contestar a las preguntas. La puntuación consiste en sumar las respuestas correctas sin tomar en cuenta los errores de ortografía. La puntuación máxima es 14 .

\section{Examen de Contenido}

Este test individual y oral fue preparado con la idea de evaluar la adquisición de las ideas que fueron articuladas en los objetivos intermedios de los programas y transmitidas mediante las actividades proporcionadas en los módulos (en el nivel de Jardín de Infancia) y en el currículum de Estudios Sociales (en los grados primero y segundo). En el nivel de Jardín de Infancia, por ejemplo, el test consistía en 22 preguntas basadas en los módulos. Aproximadamente el 50 por 100 de las preguntas se relacionaban con las series de dibujos presentados aleatoriamente al niño y que representaban animales, comidas y varios objetos. Se instruyó a los examinadores para que provocaran tanta información como fuera posible por parte del niño. A éste se le pedía que nombrara, enumerara e identificara los ítems, y que señalara las diferencias entre ellos, que nombrara ciertas caracteristicas y hechos. Si era necesario, los examinadores repetian las preguntas en inglés. El número máximo de respuestas correctas era de 69. La puntuación de cada alumno fue convertida a percentil.

Se aplicaron los tests en las clases de Jardín de Infancia Francés, Acogida y Bilingüe y en las clases correspondientes de los grados primero y segundo.

\section{Análisis de la interacción verbal.}

Esta técnica es una adaptación de la desarrollada para la clase por Flanders. Es una herramienta de observación que centra la atención del observador en la frecuencia y dirección de los intercambios verbales entre alumno y profesor y entre alumno y alumno. Los acontecimientos verbales (frases de los alumnos, preguntas, órdenes, exclamaciones) mediante los que el niño intenta comunicar sus pensamientos, se anotan en una plantilla. Puesto que sólo se observa un alumno a la vez, puede recogerse con detalle la conducta verbal y cualquier conducta paralingüística. Un observador que está familiarizado con la rutina de la clase y entrenado en el uso de la plantilla permanece cerca del alumno durante un período de diez minutos, anotando frases, con el mayor detalle posible; después se acerca a un segundo alumno.

Las observaciones fueron llevadas a cabo por dos observadores entrenados, durante el periodo en francés. Se seleccionaron ocho alumnos anglófonos en el nivel de Jardín de Infancia y fueron observados en cada otoño y primavera durante un período de tres años. En cada sesión de observación, cada alumno fue observado durante un total de veinte minutos en dos bloques de tiempo no consecutivos de diez minutos. 
La categorización de acontecimientos verbales transcritos para el análisis cuantitativo, fue llevada a cabo separadamente por dos calificadores. Había un acuerdo del 95 por 100 en la categorización de las respuestas.

\section{Cuestionario de actitudes de los alumnos}

Es un cuestionario en dos partes, administrado con el propósito de determinar de una forma global el interés del alumno, si disfruta o no en la clase, y su deseo de usar francés dentro y fuera del entorno escolar. En la Parte 1, los alumnos deben responder "sí» o "no" a frases sobre la relativa dificultad de la segunda lengua y su interés en proseguir el estudio del idioma. La parte 2 consta de una frase con una respuesta de elección múltiple en la que los alumnos pueden indicar la medida de su compromiso en el uso del idioma y su interés en usar el idioma en situaciones fuera del entorno escolar.

El cuestionario fue administrado a los alumnos del cuarto grado que habían asistido al Jardín de Infancia Bilingüe y a los alumnos del grado cuarto que asistían a las clases en escuelas que ofrecían una experiencia enriquecida de Francés como Segunda Lengua.

\section{Otros Cuestionarios (distintos de los de los alumnos)}

Se administraron un cierto número de cuestionarios, además del Cuestionario de Actitudes de los alumnos. Entre ellos están los siguientes:

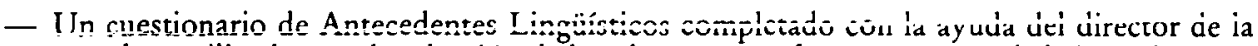
escuela y utilizado para la selección de los alumnos que formarian parte de la investigación.

- Formularios distribuidos a los dos profesores de Jardín de Infancia Bilingüe (inglés y francés) y a los dos profesores del Grado Primero (profesor de clase y especialista), pidiendo una clasificación de los alumnos en un cierto número de variables específicas de clase.

- Un cuestionario distribuido al profesor de la parte francesa del programa en el nivel de Jardín de Infancia y al profesor de Estudios Sociales en el grado primero, pidiendo comentarios sobre varios aspectos de los programas: objetivos, temas, líneas básicas, módulos, materiales de enseñanza, etc.

- Cuestionario (en forma de preguntas abiertas) para lograr una revisión general del Programa Bilingüe de Jardín de Infancia, sometido a dos especialistas, uno en el área de Jardín de Infancia (Ministerio de Educación) y otro en el área de desarrollo del lenguaje (Universidad René Descartes, París, V).

- Un Cuestionario distribuido a los padres de los niños que participaban en el programa de Jardín de Infancia Bilingüe, para evaluar sus reacciones ante el programa y ante su aplicación.

\section{B) Programa Elemental de Inmersión en Francés}

\section{Tests de Comprensión (I.O.P.F.)}

Están elaborados a partir del conjunto de tests incluidos en el Libro del Profesor de las series Ici On parle Français, nivel $2 *$. Son tests colectivos que consisten en cortas anécdotas grabadas en francés en una cinta. La anécdota se oye una vez mientras que los estudiantes escuchan. El examinador entonces para el magnetofón y distribuye copias mimeografiadas de una serie de preguntas de elección múltiple redactadas en inglés. A continuación, los estudiantes contestan a esas preguntas mientras que la anécdota se oye por segunda vez. A continuación se les da tiempo para subrayar la palabra que completa una frase de tal forma que la hace aceptable en inglés, de acuerdo con la anécdota grabada. La puntuación máxima es 10 y se obtienen puntuaciones individuales a partir del número de respuestas correctas.

* Kenney, Morgan \& Kerr, Doris: Ici On Parle Français: Teacber's Textbook. Scarborough, Ont.: Prentice-Hall ltd., 1966. 
Es un test individual grabado en cinta, tipo entrevista, que consta de las siguientes secciones:

1. Comprensión A: Ordenes e instrucciones.

El sujeto reacciona ante una serie de preguntas por medio de gestos y acciones que el examinador juzga apropiadas o inapropiadas.

2. Comprensión B: Fiases verdaderas y falsas.

El sujeto responde oui o non a una serie de frases simples.

3. Exactitud fonémica al repetir unas cuantas frases.

4. Exactitud prosódica al repetir unos cuantos patrones de entonación.

5. Expresión libre ante un dibujo que muestra la preparación de una familia para un picnic (evaluación basada en la acceptabilidad de la forma).

6. Fluidez: Evaluación basada en la eficiencia y facilidad de la comunicación.

7. Actitud hacia el aprendizaje y uso del francés según las respuestas a preguntas abiertas.

Este test fue administrado a los alumnos de Inmersión en Francés de los grados cuarto y quinto después de habérseles pasado un pretest a un grupo de niños francófonos. Las puntuaciones están basadas en criterios predeterminados y en escalas descriptivas.

\section{C) Programa de Inmersión en Francés Secundaria}

\section{Cloze Test (Inglés y Francés)}

A la luz de la investigación actual sobre la lectura, el cloze test se considera una medida excelente del nivel lingüístico global. Puesto que las pocas formas estandarizadas existentes de tests cloze no se correspondian con las características situacionales del estudio, se han desarrollado nuevos tests cloze.

Los cloze tests administrados consistían en completar un texto mutilado. El test de inglés y los dos tests de francés de aproximadamente 400 palabras de longitud fueron rigurosamente seleccionados para lograr que fueran apropiados lingüisticamente y en su contenido. Se construyeron de esta longitud con el fin de proporcionar unas cuantas frases intactas antes y después de las series de cincuenta espacios en blanco obtenidos mediante la supresión sistemática de las palabras que ocupaban el lugar séptimo. La posición del primer espacio en blanco fue decidido después de unos cuantos ensayos con una pequeña muestra de estudiantes.

El pasaje inglés "Courtesy" fue tomado de una carta circular mensual distribuida a sus clientes por un banco canadiense. Los pasajes franceses fueron tomados de dos fuentes diferentes "Louis Pasteur", tomado de una publicación periódica para adolescentes, y "La Société de consommation au Québec», de una publicación gubernamental dirigida al público en general.

Las respuestas fueron analizadas de acuerdo a un doble conjunto de criterios: semántico (exactitud, apropiación) y lingüistico (ortografía y gramática exactos). En menor medida, también según las categorias diferentes de género y uso exacto y apropiado de las preposiciones. La frecuencia de los errores fue comparada con el número de veces que aparecían las respectivas categorias lingüísticas para obtener una puntuación de porcentaje de error.

\section{Entrevista oral en francés}

Este procedimiento fue diseñado para servir a un doble propósito. Primero, se esperaba que el contenido proporcionara datos complementarios de cinco estudiantes graduados de secundaria sobre su percepción y actitudes acerca de su escolarización, particularmente en lo relacionado con el Programa Francés que se les había aplicado. Segundo, la forma lingüística proporcionaba un corpus de auténtica comunicación, que podía ser analizado y evaluado.

La entrevista consistía en una conversación grabada, semiestructurada, de veinte minutos de duración, realizada en francés. El estudiante, que podia usar el inglés cuando sintiese necesidad, tenía que responder a seis preguntas abiertas que trataban específicamente de lo siguiente:

- Cómo percibía varios aspectos del programa que se le había aplicado en comparación con otros programas. 
- Cómo se clasificaba en su nivel de inglés y en su rendimiento académico cuando se comparaba con estudiantes de otros programas.

- Qué cambios le parecería oportuno introducir y por qué.

- Qué pensaba sobre las diversas sesiones de aplicación de tests en las que había participado.

Las respuestas de los estudiantes fueron analizadas según el método ciego, desde el punto de vista del contenido y la forma, por tres estudiantes graduados de lingüística de habla francesa, que habían sido entrenados con este fin. Desde el punto de vista del contenido, se analizaron las respuestas de los estudiantes, se clasificaron y se reunieron en una rejilla paramétrica, y se convirtieron en porcentajes las frecuencias para cada pregunta, calculadas sobre la base del número total de sujetos en cada cohorte. Desde el punto de vista de la forma, las respuestas de los estudiantes se analizaron cuantitativamente de acuerdo con 21 parámetros, incluyendo el número de veces que aparecieron elementos lingüísticos específicos, y el porcentaje de los casos que se consideraban aceptables. Las respuestas de los estudiantes fueron también puntuadas según cinco parámetros cualitativos (léxico, gramatical, relativo al discurso, enunciativo y comunicativo), por medio de una escala descriptiva de siete grados. Se calcularon las medias para cada grupo de programas diferentes y se aplicó el análisis de varianza en la comparación de esas medias de grupo.

\section{Cuestionario actitudinal de los estudiantes}

Este instrumento fue ideado para que los cinco estudiantes graduados de secundaria reflejaran sus percepciones retrospectivas sobre varios aspectos de su escolarización, particularmente las relacionadas con el tipo de programa que habían escogido o al que habian sido asignados. Tiene la forma de un cuestionario con 21 preguntas cerradas y una shierta. Con !a excepciúñ de las seis que tratan de información fáctica, como idioma(s) hablado(s) en la casa, sexo, proporción de tiempo pasado en actividades francesas, etc., las otras, seis de las cuales están subdivididas en dos secciones, implican respuestas expresadas en una escala Likert. Por tanto, los estudiantes pueden indicar su evaluación personal en temas tales como:

- La conveniencia de diversos temas que podian usarse en la enseñanza indirecta del francés.

- La influencia educativa y profesional del francés.

- El grado que se atribuían a sí mismos de conocimiento del francés en comparación con el de los estudiantes que asistían a otros programas.

Las preguntas fueron reordenadas para su análisis en veintiséis variables, convirtiéndose la frecuencia de respuestas a cada pregunta en porcentajes del número total de sujetos de cada cohorte y se relacionó con cada uno de los tres grupos de comparación. El $\mathrm{X}^{2}$ resultante sirvió para determinar la significación de la diferencia entre los tres grupos comparados.

\section{Apéndice $B$}

\section{PROGRAMA DE JARDIN DE INFANCIA BILINGÜE. OBJETIVOS GENERALES (PEDAGOGICOS)}

\section{Cognitivos y educativos}

- Guiar y asistir a los niños en su adquisición de nociones elementales acerca de o sobre:

espacio (distancia, dirección, posición, etc.);

tiempo (duración, sucesión, etc.);

naturaleza (plantas, animales, etc.);

sociedad (identidad personal, familia, vecindad, etc.).

- Ayudar a los niños a derivar, a partir de su observación de los hechos, reglas de conducta personal y social. 
- Proporcionar a los niños una experiencia del idioma francés que les ayude a entender y, en su caso, a utilizar, el vocabulario básico francés y las estructuras relacionadas con el logro de los objetivos cognitivos y educativos.

3. Socio-culturales $y$ afectivos

- Ayudar a los niños a que sean conscientes de las similitudes y diferencias culturales, y desarrollen una mentalidad abierta hacia la diversidad social.

* Kenney, Morgan \& Kerr, Doris: Ici On Parle Français: Teacher's Textbook. Scarborough, Ont.: Prentice-Hall ltd., 1966.

\section{Educativos y de formación de nociones (ejemplos)}

a) - Identificar, siguiendo la observación sensorial, algunas relaciones espaciales.

- Distinguir entre varias, la colocación de personas y cosas.

- Mostrar conocimiento de las direcciones que se siguen al moverse de un sitio a otro.

- Identificar el tipo y uso de unas cuantas categorías de vehículos.

b) - Identificar las divisiones de tiempo y mostrar conocimiento de la duración.

- Mostrar cierta familiaridad con los acontecimientos climáticos relacionados temporalmente, $y$ con sus consecuencias en la vestimenta, la nutrición y las actividades generales.

- Relacionar acontecimientos espaciales con el continuo temporal: Halloween, cumpleaños, vacaciones.

\section{Lingüísticos}

a) Comprensión

- Reaccionar adecuadamente a aproximadamente 100 tipos diferentes de formulaciones de las funciones lingüísticas relacionadas con la interacción normal de una clase de jardín de infancia (p. ej., sentarse).

- Reaccionar verbalmente o por gestos a aproximadamente 50 tipos de preguntas relacionadas con la identificación, la descripción, la comparación de los fenómenos de espacio y tiempo que forman parte del contenido del programa, por ejemplo: ¿De qué color es...? ¿Cuál es el uso de...?

- Manifestar comprensión del vocabulario esencial y de la estructura usada en la presentación del contenido del programa.

- Expresar, verbalmente o de otra forma, la comprensión de las principales características de las historias relatadas por el profesor.

\section{b) Expresión}

- Utilizar apropiada y espontáneamente aproximadamente 200 palabras (léxico y función) en varios contextos de identificación.

- Conseguir un intercambio verbal relevante y natural con el profesor y los otros alumnos de acuerdo con la práctica normal en una clase de Jardín de Infancia: pedir permiso, expresar necesidades, emociones, informarse, etc.

- Describir brevemente aspectos elementales de las personas, cosas y fenómenos.

\section{Cultural}


- Recitar poemas cortos, historias o bromas.

- Dar unos cuantos nombres típicos franceses de niños, niñas, calles, etc.

\section{PROGRAMA DE ESTUDIOS (SYLLABUS)}

\section{Objetivos de contenido}

El contenido está basado en diez temas:

- Iniciación a la vida escolar.

- Relaciones sociales y actividades diarias.

- Cambios climáticos.

- Navidad.

- Animales.

- Salud y alimentos.

- Vestidos y comunidades étnicas.

- Plantas y entorno.

- Edificios y vida comunitaria.

- Transportes, viajes, vacaciones.

Cada tema se subdivide en 4 ó 5 unidades, cada una de las cuales tiene objetivos lingüísticos y conceptuales.

\section{Guia pedagógica}

Sugerencias para ayudar al profesor a interpretar el programa y a aplicarle.

\section{Guía de evaluación}

Para conseguir una evaluación formativa y sumativa.

\section{GUIAS Y MATERIALES}

Para ayudar al profesor a aplicar y evaluar el programa, se desarrollaron diez módulos, de acuerdo con el siguiente formato:

1. Tema central y subtemas.

2. Aspectos tratados en cada subtema.

3. Conjuntos específicos de formas lingüísticas que se necesitan para enseñar cada aspecto.

4. Fases diferentes en las actividades del profesor.

5. Actividades correspondientes de los alumnos y estrategias de aprendizaje.

6. Ayudas para la enseñanza: canciones, materiales, direcciones para los proyectos, referencias, catálogos, etc. 


\section{Apéndice C}

PROGRAMA DE CONTINUACION (O CURRICULUM) ESTUDIOS SOCIALES INTEGRADOS Y GRADOS PRIMERO Y SEGUNDO DE FSL

\section{OBJETIVOS GENERALES (PEDAGOGICOS)}

\section{Cognitivos y educativos}

- Guiar y asistir al niño en el posterior desarrollo de las nociones de tiempo, espacio y sociedad.

- Iniciar al niño en las estrategias científicas elementales: observación, comparación, ordenación, inferencia.

2. Lingüisticos

- Entrenar al niño para desarrollar la comprensión y el uso de las formas del idioma francés relacionadas con una interacción eficaz en la clase.

- Proporcionar al niño la oportunidad de aprender y usar el vocabulario y las estructuras francesas relacionados con el contenido del programa de estudios sociales.

3. Socioculturales $y$ afectivas

- Ayudar al niño en el posterior desarrollo de actitudes positivas hacia el aprendizaje y uso del francés y en su apertura hacia el pluralismo lingüístico y cultural.

OBJETIVOS FINALES (APRENDIZAJE)

1. Cognitivos y educativos (ejemplos)

- Concepto del espacio

a) Identificar las características naturales y artificiales (construidas por el hombre) de los alrededores locales inmediatos de cada uno.

b) Situarse a sí mismo en su entorno inmediato mediante el uso de puntos cardinales y lugares conocidos.

c) Describir cómo moverse de un sitio a otro, con el uso de un plano o mapa, indicando marcas y lugares conocidos.

d) Situar un lugar en relación con las características del entorno natural y construido por el hombre.

- Concepto del tiempo

a) Situar, en el contexto de los ciclos diarios, semanales o anuales, sus propias actividades y las de las personas de su alrededor.

b) Situar, a lo largo de un continuo temporal, los acontecimientos familiares en relación a sí mismo o a los demás.

c) Situar algunos acontecimientos en el tiempo en relación a otros acontecimientos de su propia vida o de las de los demás.

d) Describir, a partir de la observación personal, los cambios que han tenido lugar en el tiempo, en el entorno social o físico.

- Concepto de la vida en sociedad

a) Identificar varias formas mediante las que la vida familiar soluciona las necesidades materiales del niño. 
b) Identificar varias formas mediante las que la comunidad soluciona algunas otras necesidades del niño.

c) Identificar algunas características culturales y lingüísticas del propio entorno.

\section{CONOCIMIENTOS LINGÜISTICOS, EN UN CONTEXTO DE COMUNICACION EN FRANCES}

- Demostrar comprensión, mediante una respuesta verbal o física: de las peticiones de identificación o descripción de características relacionadas consigo mismo, otra persona, un grupo de personas, un objeto, un acontecimiento, un lugar; de la narración o descripción acompañada de apoyo visual de las normas relacionadas con la marcha y la interacción en la clase de los ofrecimientos de ayuda; de las concesiones o denegaciones de permiso; de las palabras de aprecio, acuerdo, reprimenda.

- Responder espontáneamente a varias clases de requerimientos.

- Contar una historia corta, o describir un acontecimiento o un fenómeno.

- Expresar, de una manera funcional, un deseo, una emoción, una condición física, una actitud, una invitación, una apreciación, un acuerdo.

- Preguntar sobre las personas, los acontecimientos, los hechos, las justificaciones.

- Expresar de una forma funcional el rechazo, el enfado, la reprimenda.

- Reproducir unas cuantas rimas y bromas.

- Cantar unas cuantas canciones francesas.

\section{OBJETIVOS SOCIOCULTURALES Y AFECTIVOS}

- Mantener una conversación efectiva con al menos una persona nativa francófona.

- Participar verbalmente en una actividad del grupo que requiera una interacción verbal en francés.

\section{OBJETIVOS Y CONTENIDOS INTERMEDIOS}

Para facilitar el gradual cumplimiento de los objetivos finales establecidos citados más arriba, se han especificado cada uno de los 11 objetivos cognitivos y educativos en tres o cuatro objetivos intermedios, incluyendo temas de estudio tanto cognitivos como lingüísticos. Para ayudar al profesor a integrar la enseñanza de los estudios sociales en francés, cada objetivo intermedio es seguido de una lista de nociones específicas y de la correspondiente terminología francesa, y de una lista de características gramaticales del francés, que deben acentuarse dentro del marco de referencia de este objetivo. Cada objetivo intermedio está formulado de tal manera que incluye actividades, materiales, tareas y procedimientos de organización. Debe hacerse notar que ni la progresión ni la limitación están rígidamente impuestas en el desarrollo de los objetivos intermedios y que, consecuentemente, la enseñanza del vocabulario específico y de las características gramaticales debe adaptarse a los conceptos o destrezas que se estén enseñando.

\section{PROGRAMA DE ESTUDIOS (SYLLABUS)}

En línea con el currículum regular, el programa de estudios experimental se ha diseñado de acuerdo con un modelo concéntrico y espiral. Los objetivos generales y finales se formulan globalmente para los primeros grados de la escuela primaria. Los conceptos, formas y destrezas que se introducen en el grado primero son gradualmente reforzados, profundizados y ampliados en los grados siguientes, mediante el uso de materiales diferenciales que han sido elaborados y coordinados a la luz de los objetivos generales, pero también con el fin de proporcionar flexibilidad.

\section{GUIAS Y MATERIALES}

Se ha hecho una relación de una serie de precauciones y sugerencias, insistiendo en la prioridad de la metodología y contenido que debe darse a la enseñanza de los estudios sociales, en 
el uso exclusivo del francés como idioma de instrucción, en la flexibilidad de la tarea y las actividades que se requieren $y$ en la necesidad de adaptarse al ritmo de aprendizaje del niño $y, a l$ mismo tiempo, a la necesidad de mostrar creatividad en la estimulación del aprendizaje. La atención del profesor se dirige a la importancia de idear maneras y medios de proporcionar un uinput comprensible» (nocional y lingüístico), sin recurrir al inglés o a un lenguaje no natural. Se recuerda al profesor que utilice la discrección y la estimulación para motivar al niño a incrementar su uso del francés en su interacción con él. Finalmente, se le dan al profesor unas pocas normas de guía en el uso de materiales básicos, de referencia, y publicaciones.

Se recomienda usar como materiales básicos las series llamadas Collection Eveil à la réalité (Colección Despertar a la realidad) (1978), que han sido elaboradas para la enseñanza de estudios sociales en los tres primeros grados de la escuela primaria. El autor, Jean-Luc Picard proporciona, para cada nivel, un manual del profesor y un libro de ejercicios del alumno. La selección y progresión del contenido son compatibles con los objetivos finales de programa experimental, pero requieren adaptación por parte del profesor en relación con la formulación lingüística. Los tres libros son los siguientes:

Grado primero: Autour de moi (Lo que me rodea).

Grado segundo: Moi et les miens (Yo y los míos).

Grado tercero: En passant par chez nous (En nuestra casa).

Los libros de texto básicos proporcionan actividades de evaluación que pueden usarse en la evaluación formativa y sumativa, pero se le dan al profesor unas cuantas sugerencias prácticas con el fin de adaptar esas actividades y tareas, y también unos cuantos principios que deben seguirse en la preparación y uso de sus propios instrumentos.

\section{Apéndice a (Resultados)}

TENDENCIAS GLOBALES EN LOS RESULTADOS EN LOS TETS DE LENGUA INGLESA

JARDIN DE INFANCIA Y SU CONTINUACION

Grupos:

\begin{tabular}{|c|c|c|c|}
\hline Jardin de inf̣an. & Grado 1 & Grado 2 & Grado 3 \\
\hline $\begin{array}{l}\text { Exp. }=R .=I R \\
\text { en Raven } \\
\text { Peabody } \\
\text { OLM }\end{array}$ & $\begin{array}{l}\text { Exp. }=R .=I R \\
\text { en Raven } \\
\text { Peabody } \\
\text { OLM }\end{array}$ & $\begin{array}{l}\text { Exp. }=R .=I R \\
\text { en Raven } \\
\text { MAT } \\
\text { Lect. }\end{array}$ & $\begin{array}{l}\text { Exp. }=R .=I R \\
\text { en MAT } \\
\text { Lect. } \\
\text { Leng. }\end{array}$ \\
\hline
\end{tabular}

$\begin{array}{ll}\text { Exp. } & =\text { Experimental (Grupo) } \\ \text { R. } & =\text { Recepción } \\ \text { IR } & =\text { Inglés Regular }\end{array}$

INMERSION ELEMENTAL

$\begin{array}{ccc}\text { Grado } 4 & \text { Grado 5 } & \text { Grado 6 } \\ (90 \% \text { FR }) & (90 \% \text { FR }) & (50 \% \text { FR })\end{array}$

\begin{tabular}{llll}
\hline \multicolumn{1}{c}{ Pretest } & \multicolumn{1}{c}{ Posttest } & \multicolumn{1}{c}{ Posttest } & \multicolumn{1}{c}{ Posttest } \\
\hline IE = IR & IE = IR & IE =IR & IE = IR \\
en CTBS & en CTBS & en CTBS & en CTBS \\
Voc. & Voc. & Voc. & Voc. \\
Lect. & Lect. & Lect. & Lect. \\
Leng. & Punt. & IR = IE & Leng. \\
Mayusc. & IR = IE & Mayusc. & Mayusc. \\
Punt. & Ortografía & Punt. & Punt. \\
en Raven & Mayusc. & Ortografía & \\
en Lorge-Th. & & & \\
\hline
\end{tabular}

IE $\quad=$ Inmersión Elemental

IR = Inglés Regular 


\begin{tabular}{lll}
\hline \multicolumn{1}{c}{ Grado 7 (Sec. 1) } & \multicolumn{1}{c}{ Grado 8 (Sec. 2) } & \multicolumn{1}{c}{ Grado 9 (Sec. 3) } \\
\hline IPE IFS IR & IPE IFS IR & IPE IFS IR \\
en MAT & en MAT & en MAT \\
Conoc. Mundo & Conoc. Mundo & Conoc. Mundo \\
Lect. & Lect. & Lect. \\
Leng. & Leng. & Leng. \\
Ortografia & Ortografía & Ortografia \\
en Kaven & en Raven & en Raven \\
Lorge-Th & Lorge-Th & Lorge-Th \\
\hline
\end{tabular}

IPE = Inmersión Post-Elemental

IFS = Inmersión Francés Secundaria

IR = Inglés Regular

\section{Apéndice b (Resultados)}

TENDENCIAS GLOBALES DE LOS RESULTADOS EN LOS TESTS DE LENGUA FRANCESA JARDIN DE INFANCIA BILINGÜE Y SU CONTINUACION

\begin{tabular}{|c|c|c|c|}
\hline Jardin de Infancia & Grado 1 & Grado 2 & Grado 3 \\
\hline $\begin{array}{l}\text { FR }=R=\text { Exp. } \\
\text { en Peabody }(F r) \\
\text { ESB }\end{array}$ & $\begin{array}{l}\mathrm{R}=\mathrm{Exp} .=\mathrm{IR} \\
\text { en OSIE } \\
\text { OLM (Fr) }\end{array}$ & $\begin{array}{l}\mathrm{R}=\mathrm{Exp} .=\mathrm{IR} \\
\text { en Peabody (Fr) } \\
\text { OLM (Fr) }\end{array}$ & $\begin{array}{l}\quad R=\text { Exp.=IR } \\
\text { en OISE } \\
\text { OLM (Fr) } \\
\text { B\&G (Lect.) }\end{array}$ \\
\hline $\begin{array}{l}\mathrm{R}=\mathrm{Exp} \\
\text { OISE } \\
\text { OLM (Fr) }\end{array}$ & $\begin{array}{l}\mathrm{R}=\text { Exp. } \\
\text { en Content }\end{array}$ & $\begin{array}{l}\mathrm{R}=\text { Exp. } \\
\text { en Content }\end{array}$ & Docu (Lel.) \\
\hline
\end{tabular}

$$
\begin{array}{ll}
\text { Exp. } & =\text { Experimental } \\
\mathrm{R} & =\text { Reception } \\
\mathrm{FR} & =\text { Francés Regular } \\
\mathrm{IR} & =\text { Inglés Regular }
\end{array}
$$

INMERSION FRANCES ELEMENTAL

\begin{tabular}{lll}
\hline \multicolumn{1}{c}{ Grado 4 } & \multicolumn{1}{c}{ Grado 5 } & \multicolumn{1}{c}{ Grado 6 } \\
\hline $\begin{array}{l}\text { IE=IR } \\
\text { en Test de Compr. (OIPF) } \\
\text { Compr. y Exp. Orales }\end{array}$ & $\begin{array}{l}\text { IE=IR } \\
\text { un Compr. y Exp. Orales }\end{array}$ & $\begin{array}{l}\text { FR=IE } \\
\text { en tests de L. (SLP) } \\
\text { tests de L. Silenc. } \\
\text { tests de L. Calif. }\end{array}$ \\
$\begin{array}{l}\text { FR=IE=IR } \\
\text { en Je Sais }\end{array}$ & $\begin{array}{l}\text { FR=IE=IR } \\
\text { en Je sais } \\
\text { en prueba (SLP) }\end{array}$ \\
& $\begin{array}{l}\text { Tests de L. Calif. } \\
\text { CECM } \\
\text { FR=IE }=\end{array}$ \\
& & \\
& IR \\
& en Je Sais & \\
\hline
\end{tabular}

IE $\quad=$ Inmersión Elemental 
INMERSION FRANCES SECUNDARIA

\begin{tabular}{|c|c|c|c|c|}
\hline Grado 7 & Grado 8 & Grado 9 & Grado 10 & Grado 11 \\
\hline $\begin{array}{l}\text { FR PIE IRS IS IR } \\
\text { en Test de Lect. } \\
\text { (SLP) }\end{array}$ & $\begin{array}{l}\text { PIE IS } \\
\text { en CECM (FR) }\end{array}$ & $\begin{array}{l}\text { FR PIE IS IR } \\
\text { en Test de } \\
\text { Lect. (SLP) }\end{array}$ & $\begin{array}{l}\text { PIE IS } \\
\text { en FR. Cloze }\end{array}$ & \multirow{2}{*}{$\begin{array}{l}\text { PIE IS } \\
\text { En actitudes hacia } \\
\text { el Progr. Fran. } \\
\text { Auto-evaluación } \\
\text { PIE IS } \\
\text { en Actitudes hacia } \\
\text { uso del francés }\end{array}$} \\
\hline $\begin{array}{l}\text { IR PIE IS IRS IR } \\
\text { en Test de Lect. } \\
\text { (Calif.) }\end{array}$ & $\begin{array}{l}\text { PIE IS } \\
\text { en CECM (Math) } \\
\text { en Comp. Fr. }\end{array}$ & $\begin{array}{l}\text { PIE IS } \\
\text { n)en Test de } \\
\text { Lect. (Calif) }\end{array}$ & $\begin{array}{l}\text { PIE IS } \\
\text { en Comp. Fr. } \\
\text { el aprendizaje y }\end{array}$ & \\
\hline $\begin{array}{l}\text { FR PIE IS } \\
\text { en CECM } \\
\text { PIE IS } \\
\text { en Composición Fr. }\end{array}$ & & $\begin{array}{l}\text { FR PIE IS } \\
\text { en CECM (Fr) } \\
\text { PIE IS } \\
\text { en Composición Fr. }\end{array}$ & & $\begin{array}{l}\text { PIE IS IR } \\
\text { en Cloze Ingl. } \\
\text { PIE IS IR } \\
\text { en Cloze Fr. }\end{array}$ \\
\hline
\end{tabular}

PIE = Post Inmersión Elemental (Grupo).

IS = Inglés Secundaria.

IRS = Inglés Regular Secundaria.

IR = Inglés Regular (a partir del grado cuarto).

$\mathrm{FR} \quad=$ Francés Regular. 